\title{
Arabidopsis R2R3-MYB transcription factor AtMYB60 functions as a transcriptional repressor of anthocyanin biosynthesis in lettuce (Lactuca sativa)
}

\author{
Jong-Sug Park $\cdot$ Jung-Bong Kim $\cdot$ Kang-Jin Cho $\cdot$ \\ Choong-Ill Cheon $\cdot$ Mi-Kyung Sung · \\ Myoung-Gun Choung · Kyung-Hee Roh
}

Received: 18 November 2007/Revised: 30 January 2008/Accepted: 17 February 2008/Published online: 4 March 2008

(C) The Author(s) 2008

\begin{abstract}
The MYB transcription factors play important roles in the regulation of many secondary metabolites at the transcriptional level. We evaluated the possible roles of the Arabidopsis R2R3-MYB transcription factors in flavonoid biosynthesis because they are induced by UV-B irradiation but their associated phenotypes are largely unexplored. We isolated their genes by RACE-PCR, and performed transgenic approach and metabolite analyses in lettuce (Lactuca sativa). We found that one member of this protein family, AtMYB60, inhibits anthocyanin biosynthesis in the lettuce plant. Wild-type lettuce normally accumulates anthocyanin, predominantly cyanidin and traces of delphinidin, and develops a red pigmentation. However, the production and
\end{abstract}

Communicated by J.R. Liu.

J.-S. Park · J.-B. Kim · K.-J. Cho · K.-H. Roh $(\bowtie)$

Molecular Physiology and Biochemistry Division,

National Institute of Agricultural Biotechnology,

Suwon 441-707, South Korea

e-mail: rohkh@rda.go.kr

J.-S. Park

e-mail: jongsug@rda.go.kr

C.-I. Cheon

Department of Life Science,

Sookmyung Women's University,

Youngsan-gu, Seoul 140-742, South Korea

M.-K. Sung

Department of Food and Nutrition,

Sookmyung Women's University,

Youngsan-gu, Seoul 140-742, South Korea

M.-G. Choung

Department of Pharmacognosy Material Development,

Kangwon National University,

Samcheok 245-711, South Korea accumulation of anthocyanin pigments in AtMYB60-overexpressing lettuce was inhibited. Using RT-PCR analysis, we also identified the complete absence or reduction of dihydroflavonol 4-reductase (DFR) transcripts in AtMYB60- overexpressing lettuce (AtMYB60-117 and AtMYB60-112 lines). The correlation between the overexpression of AtMYB60 and the inhibition of anthocyanin accumulation suggests that the transcription factorAtMYB60 controls anthocyanin biosynthesis in the lettuce leaf. Clarification of the roles of the AtMYB60 transcription factor will facilitate further studies and provide genetic tools to better understand the regulation in plants of the genes controlled by the MYB-type transcription factors. Furthermore, the characterization of AtMYB60 has implications for the development of new varieties of lettuce and other commercially important plants with metabolic engineering approaches.

Keywords R2R3-MYB transcription factor - Repressor · Arabidopsis $\cdot$ Lactuca sativa

$\begin{array}{ll}\text { Abbreviations } \\ R T-P C R & \begin{array}{l}\text { Reverse transcription-polymerase chain } \\ \text { reaction }\end{array} \\ R A C E- & \begin{array}{l}\text { Rapid amplification of cDNA ends-polymerase } \\ \text { chain reaction }\end{array} \\ P C R & \text { Cauliflower mosaic virus } \\ C a M V & \text { Chalcone synthase } \\ C H S & \text { Dihydroflavonol 4-reductase } \\ D F R & \text { Chalcone isomerase } \\ C H I & \text { Flavanone 3-hydroxylase } \\ F 3 H & \text { Anthocyanidin synthase } \\ A N S & \text { UDP-glucose:flavonoid } \\ U F G T & \text { 3-O-glucosyl } \\ & \text { transferase }\end{array}$




\section{Introduction}

Flavonoid metabolism is unique to plants and produces a great number and variety of compounds including flavones, flavonols, anthocyanins, and proanthocyanins (WinkelShirley 2001; Park et al. 2004). These molecules play important roles in many fundamental processes in plants, such as the pigmentation of flowers and fruits, UV resistance, pollen fertility, responses to wounding and fungal attacks, and environmental stress responses (Shirley et al. 1995; Weisshaar and Jenkins 1998; Harborne and Williams 2000; Dixon and Piava 1995; Moyano et al. 1996; Pietta 2000; Cominelli et al. 2005). The regulation of the anthocyanin pigments in plants is particularly important in metabolic engineering applications because these compounds act as visual signals that attract the insects and animals required for pollination and seed dispersal (Holton and Cornish 1995).

The metabolic pathways involved in anthocyanin biosynthesis are well established, and the central pathways in this process are highly conserved in plants (Shih et al. 2006). Moreover, studies in model plants, such as Arabidopsis and maize, have facilitated a sound understanding of the genes involved and the assembly of the relevant enzyme complexes (Winkel-Shirley 2001). Two groups of genes are required for anthocyanin biosynthesis: structural genes that encode the enzymes that directly participate in the biosynthetic reactions, and genes for transcription factors that regulate the expression of these structural genes and the accumulation of flavonoid metabolites. Transcription factors can act as activators or repressors of gene expression, and mediate either increases or decreases in transcription through sequence-specific DNA binding and protein-protein interactions (Broun 2004).

MYB proteins have been identified in a large number of eukaryotes, including fungi, plants and vertebrates (Ohi et al. 1994; Martin and Paz-Ares 1997; Slamon et al. 1986), and have roles in a wide array of cellular processes. These include the regulation of secondary metabolism, signal transduction, cell division, and responses to plant diseases and various forms of stress (UV-B light, cold and drought) (Larkin et al. 1994; Borevitz et al. 2000; Stracke et al. 2001; Vailleau et al. 2002; Cominelli et al. 2005). The R2R3-MYB-related proteins activate the transcription of structural genes that function in different branches of phenylpropanoid metabolism (Martin and Paz-Ares 1997). The promoters of these structural genes each contain potential MYB protein recognition motifs and also bind with the basic helix-loop-helix (bHLH) domain and conserved WD40 repeat proteins (Park et al. 2007a, b; Martin and Paz-Ares 1997; Winkel-Shirley 2001).
The classification of MYB transcription factors is based on the strong conservation of imperfect repeats in the MYB DNA-binding domain of the member proteins (Martin and Paz-Ares 1997). In contrast to animals (R1R2R3), the MYB domain transcription factors found in plants are characterized by the R2R3-type MYB domain, comprising a DNA-binding domain and an activation/repression domain. Moreover, the balance between activators and repressors in this transcription factor family in plants may provide extra flexibility in terms of transcriptional control (Jin et al. 2000). There have been 126 R2R3 MYB genes identified in Arabidopsis thaliana, all of which exhibit strong homology within their N-terminal MYB DNAbinding domains and can be divided into 24 subgroups on the basis of their sequences (Stracke et al. 2001; Riechmann and Ratcliffe 2000; Kranz et al. 1998). To determine the biological roles of the MYB-related transcription factors in the production of phenylpropanoids, we isolated six R2R3-MYB proteins from Arabidopsis based on available information indicating that these genes are induced by environmental stress (UV-B irradiation) (Kranz et al. 1998). However, they do not have an informative phenotype, because of the structural and functional redundancy among these factors (Bouche and Bouchez 2006). Furthermore, although there have been other studies of anthocyanin accumulation in lettuce, one of the most popular and commercially important vegetables in the world, this process has not been characterized before at the level of transcriptional regulation. The regulation of anthocyanin accumulation in plants is also a key issue in our understanding of the regulation of leaf color. The identification of the factors that exert this control will provide valuable tools to moderate the extent and distribution of anthocyanin-derived pigmentation in plant tissues.

In this study, we overexpressed the AtMYB60 gene in lettuce plants ("Jinjachuckmyun" cultivar) that are highly pigmented with a red color throughout their leaf tissues. Because the loss of these pigments is easily detected, the activity and repression of the MYB-type transcription factors that affect these pathways can be investigated. In the leaves of lettuce, coordinated transcriptional regulation controls virtually each step of the anthocyanin biosynthetic pathway (Park et al. 2007a). We compared the expression of the major anthocyanin biosynthetic genes and the AtMYB60 gene by RT-PCR. We show that this transcription factor is functionally active in repressing anthocyanin accumulation and can thus generate green lettuce leaves. We also show, by functionally repressing the accumulation of this compound, that $A t M Y B 60$ plays a significant role in controlling anthocyanin biosynthesis via the inhibition of a key target gene encoding dihydroflavonol reductase (DFR). 
Thus, we report here for the first time that anthocyanin biosynthesis and the transcription of the DFR gene are repressed through the overexpression of Arabidopsis R2R3-MYB transcription factors in transgenic lettuce plants. We also discuss the function of transcriptional regulators in the control of the expression of the structural protein that are required for anthocyanin biosynthesis in lettuce.

\section{Materials and methods}

Plant materials and growth condition

To investigate the effects of exogenous AtMYB proteins on anthocyanin biosynthesis in lettuce leaves at the molecular level, Jinjachuckmyun plants, supplied by Syngenta (Seoul, Korea), were used. The plants were grown at the National Institute of Agricultural Biotechnology in Suwon, South Korea. Fresh lettuce leaves were plucked from the plants, frozen immediately in liquid nitrogen and stored at $-80^{\circ} \mathrm{C}$ until required.

\section{Isolation of $A t M Y B$ cDNAs}

To isolate the AtMYB genes, cDNAs were isolated from UV-B-irradiated Arabidopsis plants using the BD SMART RACE cDNA Amplification Kit (Clontech, USA) and then used as templates for PCR cloning. The following genespecific primers were used for RACE-PCR: AtMYB4 (forward: 5'-GTATGGGAAGGTCACCGTGCTGTGAGAAA3'; reverse: 5'-TATTATTTCATCTCCAAGCTTCGAAA GCC-3'), AtMYB29 (forward: 5'-AAATGTCAAGAAAGC CATGTTGTGTGGGA- ${ }^{\prime}$; reverse: $5^{\prime}$-GATCATATGAAG TTCTTGTCGTCATAATC-3'), AtMYB30 (forward: 5'-TA ATGGTGAGGCCTCCTTGTTGTGACAAA- $3^{\prime}$; reverse: 5'-CTTCAGAAGAAATTAGTGTTTTCATCCAA- ${ }^{\prime}$ ), AtMYB34 (forward: 5'-AGATGGTGAGGACACCATGTT GCAAAGAA- $3^{\prime}$; reverse: 5'-CGTCAGACAAAGACTCC AACCATATTGTC-3'), AtMYB51 (forward: 5'-GAATGGT GCGGACACCGTGTTGCAAAGCC- $3^{\prime}$; reverse: $5^{\prime}$-AC TCATCCAAAATAGTTATCAATTTCGTC- $3^{\prime}$ ) and AtMYB60 (forward: 5'-AGATGGGTAGGCCTCCATGCT GTGACAAG- ${ }^{\prime}$; reverse: $5^{\prime}$-AATTAAAGCATATTAGAGAGCTCCATCAA- $3^{\prime}$ ). The resulting PCR products were then cloned into the pGEM-T Easy vector (Promega, USA) and sequenced on both strands.

Vector construction

$A t M Y B$ coding regions were cloned into an expression vector containing the CaMV $35 \mathrm{~S}$ promoter and the nopaline synthase $3^{\prime}$ terminator. The resulting constructs were then introduced into the binary vector 22103 (a derivative of pCAMBIA3301; Cambia, Australia). The complete coding sequence of each $A t M Y B$ cDNA was amplified with a specific forward primer designed to introduce an $M l u \mathrm{I}$ restriction site and a reverse primer designed to introduce an $X b a \mathrm{I}$ restriction site to allow subcloning, as follows: AtMYB4 (forward: 5'-ATACGCGTATGGGAAGGTCACC GTGCTGTG-3'; reverse: 5'-TATCT AGATTATTTCAT CTCCAAGCTTCGA-3'), AtMYB29 (forward: 5'-ATACG CGTATGTCAAGAAAGCCATGTTGTG-3'; reverse: $5^{\prime}$ TATCTAGATCATATGAAGTTCTTGTCGTCA-3'), AtMYB30 (forward: 5'-ATACGCGTATGGTGAGGCCTC CTTGTTGTG- $3^{\prime}$; reverse: $5^{\prime}$-TATCTAGATCAGAAGAA ATTAGTGTTTTCA-3'), AtMYB34 (forward: 5'- ATACGCGTATGGTGAGGACACCATGTTGCA- $3^{\prime}$; reverse: 5'-TATCTAGATCAGACAAAGACTCCAACCATA-3'), AtMYB51 (forward: 5'-ATACGCGTATGGTGCGGACA CCGTGTTGCA-3'; reverse:5'-TATCTAGATCATCCAA AATAGTTATCAATT- $3^{\prime}$ ), AtMYB60 (forward: 5'-ATACGCGTATGGGTAGGCCTCCATGCTGTG-3'; reverse: 5'-TATCTAGATTAAAGCATATTAGAGAGCTCC-3').

After PCR and digestion, the $M l u \mathrm{I} / \mathrm{X} b a \mathrm{I}$ fragments were cloned into the binary vector 22103 between CaMV 35S promoter and the nopaline synthase poly(A) addition site, creating the binary vector 22103-AtMYB plasmids (Fig. 2). These constructs were then introduced into the Agrobacterium tumefaciens EHA105 strain using the freeze-thaw method.

\section{Generation of AtMYB transgenic lettuce}

Transgenic lettuce plants were generated using the Agrobacterium-mediated transformation of cotyledons. Lettuce seeds of the Jinjachuckmyun cultivar were supplied by Syngenta (Korea) and were surface sterilized in $25 \%(\mathrm{v} / \mathrm{v})$ bleach for $7 \mathrm{~min}$, washed (three changes) in sterile water, and placed on phytogel-solidified $(0.2 \%$, w/v) MS medium at $\mathrm{pH}$ 5.8. The seeds were germinated and maintained at $25^{\circ} \mathrm{C}\left(16 \mathrm{~h}\right.$ photoperiod, $200 \mu \mathrm{mol} \mathrm{m}{ }^{-2} \mathrm{~s}^{-1}$, daylight fluorescent tubes). The cotyledons were excised from the 4day-old lettuce seedling and inoculated with freshly grown A. tumefaciens. The inoculated cotyledons were then cocultivated on MS salt medium solidified with $0.2 \%$ purified phytogel (Sigma, USA), for 2 days at $24^{\circ} \mathrm{C}$ in the dark. The cotyledon explants were then transferred to shoot initiation medium supplemented with $400 \mathrm{mg}^{-1}$ carbenicillin (Duchefa, the Netherlands). After a washing step, these explants were cultured (ten per plate) on selection medium (MS salt $4.43 \mathrm{~g} \mathrm{l}^{-1}$, sucrose $30 \mathrm{~g}^{-1}$, phytogel $2 \mathrm{~g} \mathrm{l}^{-1}$, kinetin $0.5 \mathrm{mg} \mathrm{l}^{-1}$, carbenicillin $400 \mathrm{mg} \mathrm{l}^{-1}$, phosphinothricin $1.0 \mathrm{mg}^{-1}$ and cysteine $10 \mathrm{mg} \mathrm{l}^{-1}$ ) and subcultured every 2 weeks on the same medium. Shoots that regenerated from explants on medium containing phosphinothricin 
were rooted in rooting medium (MS salt $4.43 \mathrm{~g} \mathrm{l}^{-1}$, sucrose $30 \mathrm{~g} \mathrm{l}^{-1}$, phytogel $2 \mathrm{~g} \mathrm{l}^{-1}$ and phosphinothricin $1.0 \mathrm{mg} \mathrm{l}^{-1}$ ), before transfer to the greenhouse, where they were allowed to self-pollinate and to set seed. The seeds were harvested and stored at $4{ }^{\circ} \mathrm{C}$.

Anthocyanin extraction and HPLC analysis

Anthocyanin in the lettuce plants was quantified by HPLC at a detection wavelength of $510 \mathrm{~nm}$, comparing the sample retention times and peaks with those of known standards. The relative quantification of each phenolic compound samples was expressed as the equivalent quantity of purified standards for each treatment.

About $0.2 \mathrm{~g}$ of ground and freeze-dried leaves was accurately weighed and extracted with $1 \mathrm{ml}$ of a solution of methanol:water $(80: 20, \mathrm{v} / \mathrm{v})$ at $80^{\circ} \mathrm{C}$ for $1 \mathrm{~h}$, then sonicated for $20 \mathrm{~min}$. The extracted solution was filtered into a $50-\mathrm{ml}$ volumetric flask, and the flask and filter were rinsed with a solution of methanol:water $(80: 20, \mathrm{v} / \mathrm{v})$. The filtrate was then made up to the required volume with the same solvent. Approximately $1 \mathrm{~mL}$ of the sample solution was passed through a $0.4 \mu \mathrm{m}$ filter before analysis by HPLC. A Shimadzu HPLC system with 10AD dual pumps was used, with an RP18 (LiChrospher, $250 \mathrm{~mm} \times 4 \mathrm{~mm} \times 5 \mu \mathrm{m}$ ) column. The HPLC parameters were as follows: column temperature $30^{\circ} \mathrm{C}$, solvent $\mathrm{A}=0.1 \%$ trifluoroacetic acid in water, solvent $\mathrm{B}=98 \%$ acetonitrile with $0.1 \%$ trifluoroacetic acid, solvent gradient, $0 \mathrm{~min}=0 \% \mathrm{~B}, 3 \mathrm{~min}=6 \%$ $\mathrm{B} 12 \min =18 \% \mathrm{~B}, 25 \min =25 \% \mathrm{~B}, 35 \mathrm{~min}=100 \% \mathrm{~B}$, $40 \mathrm{~min}=100 \% \mathrm{~B}$. The flow rate was $0.35 \mathrm{~mL} \mathrm{~min}^{-1}$. The peaks were classified as either cyanidin or delphinidin derivatives by UV spectral analysis.

\section{RT-PCR analysis}

Total RNA from the transgenic and wild-type lettuce leaves was extracted using RNeasy Plant Mini Kit (Qiagen, USA), according to the manufacturer's instructions. To estimate the transcript levels of the exogenous AtMYB4 and AtMYB60 genes in lettuce, we used the primers 5'-ATGG GAAGATCGCCTTGTTGTGAA- $3^{\prime}$ (F) and 5'-TCATTTC ATCTCTAAGCTTCTGTAGTCCAAAA- $3^{\prime}$ (R) for $A t$ MYB4 and $5^{\prime}$-ATGGGGAGGCCTCCTTGTTGTG-3' (F) and $5^{\prime}$-TCAATTATCGAAAAAATTAGGGTTTTCATC A-3' (R) for AtMYB60. The mRNA expression patterns of the $C H S, F 3 H, D F R$ and $U F G T$ genes were analyzed by RT-PCR with the All-in-one RT/PCR Premix (SuperBio P7003, Suwon, Korea). The amplification of rRNA was used as the internal control. Total RNA (100 ng) in a volume of $20 \mu \mathrm{L}$ containing $20 \mathrm{mM}$ Tris- $\mathrm{HCl}(\mathrm{pH} 7.9)$, $100 \mathrm{mM} \mathrm{KCl}, 0.1 \mathrm{mM}$ EDTA, $1 \mathrm{mM}$ DTT, M-MLV reverse transcriptase (RNaseH Minus), Super Taq Plus
DNA polymerase and $10 \mathrm{pmol}$ of each gene-specific amplification primer was used for RT-PCR, according to the manufacturer's instructions. Gene-specific primers for CHS (forward: 5'-GGTTTGCTCTGAGATTACAGCGG TTACC- $3^{\prime}$, reverse: $5^{\prime}$-TCCTTGAGACCAAGCTTGAGC TCCACCT-3'), $F 3 H$ (forward: $5^{\prime}$-GAGATCTTATCAGA GGCAATGGGCCTTG- $3^{\prime}$, reverse: $5^{\prime}$-ACAACGGCCCG ATGGTCTGCGTTCTTAA- $3^{\prime}$ ), DFR (forward: $5^{\prime}$-GGTCT ATGACGAGTCTCATTGGAGCGAT- ${ }^{\prime}$, reverse: $5^{\prime}$-CTA TCAATTGCTCCTTTGAACATCTCCT- ${ }^{\prime}$ ), $U F G T$ (forward: 5'-TGGAGAAGCGGGTTAGACAGTTGATGGA$3^{\prime}$, reverse: 5'-TATAGCTACCATGATTCAACCAACTT CG-3'), $r R N A$ (forward: 5'-TACGGCACTGAAGGTGCC AAGCTCGTG-3', reverse: 5'-CATCCTCTTGGCAGTCT TGGCGTAGGG-3') and bar (forward 5'-GCCGCAGGA ACCGCAGGAGT- $3^{\prime}$, reverse: 5'-AGCCCGATGACAG CGACCAC- $3^{\prime}$ ) were used to confirm the expression levels of these genes in the lettuce plants. Reverse transcription of the total RNA was carried out at $50^{\circ} \mathrm{C}$ for $30 \mathrm{~min}$, and the reaction was inactivated at $96^{\circ} \mathrm{C}$ for $3 \mathrm{~min}$. The amplification conditions comprised 35 cycles of denaturation at $94^{\circ} \mathrm{C}$ for $30 \mathrm{~s}$, annealing at $60^{\circ} \mathrm{C}$ for $30 \mathrm{~s}$, extension at $72^{\circ} \mathrm{C}$ for $2 \mathrm{~min}$, and a final extension at $72^{\circ} \mathrm{C}$ for $10 \mathrm{~min}$. PCR was performed using a GeneAmp PCR system 9700 Cycler (Perkin-Elmer, MA, USA). The resultant RT-PCR products were resolved on a $1 \%$ agarose gel, stained with ethidium bromide, and photographed. The amplified products of the CHS, F3H, DFR, UFGT, rRNA and bar transcripts were $398,319,534,153,386$ and $267 \mathrm{bp}$, respectively.

\section{Results}

Cloning of MYB-type transcription factor genes from Arabidopsis

To isolate and characterize the MYB-type transcription factors in Arabidopsis (AtMYB) that play a role in phenylpropanoid metabolism, primers were designed to amplify the entire coding regions of the AtMYB genes that are induced by environmental stress (UV-B irradiation) (Meissner et al. 1999). Previous studies of Arabidopsis have suggested that the R2R3-MYB genes of this plant have important functions in the regulation of secondary metabolism, disease resistance, and hormonal responses (Kranz et al. 1998). We isolated six Arabidopsis R2R3MYB transcription factor genes based on available information concerning the $A t M Y B$ genes induced by UV-B irradiation (Kranz et al. 1998). These were AtMYB4 (AF062860, AT4G38620), AtMYB29 (AF062872, At5G07690), AtMYB30 (AF062873, AT3G28910), AtMYB34 (U66462, AT5G60890), AtMYB51 (AF062887, 
AT1G18570) and AtMYB60 (AF062895, AT1G08810), with apparent full-length cDNAs of 849, 1011, 972, 885, 1059 and $843 \mathrm{bp}$, respectively, encoding proteins of 283 , 337, 324, 295, 353 and 281 amino acids, respectively (Fig. 1). The amino termini of these proteins contain the R2R3 repeats responsible for their binding to target DNA sequences and which are highly conserved among the R2R3-MYB proteins.

\section{Expression of $A t M Y B$ genes in lettuce plants}

To examine the effects of the six isolated $A t M Y B$ genes on leaf color changes and on the anthocyanin composition of lettuce, cotyledons of the cultivar "Jinjachuckmyun" were transformed using the Agrobacterium tumefaciens strain EHA105 with the binary vector 22103-AtMYB (a derivative of pCAMBIA3301; Fig. 2), carrying these genes and a barstar selection marker. Shoots were regenerated from the callus of phosphinothricin-resistant transformatants. To confirm the introduction and expression of the exogenous $A t M Y B$ genes in the Jinjachuckmyun cultivars, total RNA was extracted from the leaves of six-week-old wild-type and transformed lettuce plants (T1 generation) and analyzed by RT-PCR.

Transgenic plants were confirmed by their resistance to a barstar spray and by subsequent RT-PCR with specific primers for six AtMYB genes. Because the T1 plants of the AtMYB4, AtMYB29, AtMYB30, AtMYB34 and AtMYB51 transformants were similar in leaf color and in the anthocyanin accumulation in their leaves, the AtMYB4

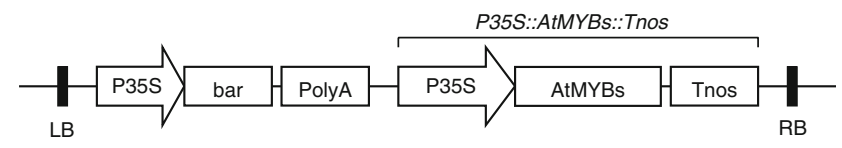

Fig. 2 Schematic diagram of the T-DNA region of the 22103AtMYB binary vector used in the transformation of lettuce cultivars. $L B$ left border, $R B$ right border, $P 35 S$ Cauliflower mosaic virus $35 \mathrm{~S}$ RNA promoter, PolyA Cauliflower mosaic virus $3^{\prime}$ UTR terminator, Tnos nopaline synthase terminator

transformant (AtMYB4-101) and the AtMYB60 transformant (AtMYB60-117 and AtMYB60-112) were selected for further RT-PCR analysis to detect the expression of the structural genes that are involved in anthocyanin biosynthesis (Fig. 3). RT-PCR analysis was performed using sixweek-old lettuce leaves of wild-type and transgenic lettuce plants. The wild-type lettuce plants had no integrated bar gene whereas each of the transgenic plants expressed bar mRNA. Analysis of the exogenously expressed AtMYB4 and $A t M Y B 60$ genes showed that $A t M Y B 4$ was specifically expressed in AtMYB4-101 and AtMYB60 in AtMYB60117 and AtMYB60-112. Ribosomal RNA levels were used as a normalization control.

\section{Plant morphology in $A t M Y B$-transformed lettuce}

In our previous study, we demonstrated that the wild-type lettuce leaf accumulates anthocyanin, with cyanidin and delphinidin representing the major and minor components, respectively (Park et al. 2007a). To further characterize the functions of the AtMYB genes in anthocyanin biosynthesis,
Fig. 1 Multiple alignment of AtMYB proteins. Amino acids identical in all six proteins are marked in black, amino acids found in four proteins are marked in gray. The line above indicates the R2 and R3 MYB repeats. GenBank accession numbers for the sequences: AtMYB4 (AF062860), AtMYB29 (AF062872), AtMYB30 (AF062873), AtMYB34 (U66462), AtMYB51 (AF062887) and AtMYB60 (AF062895)

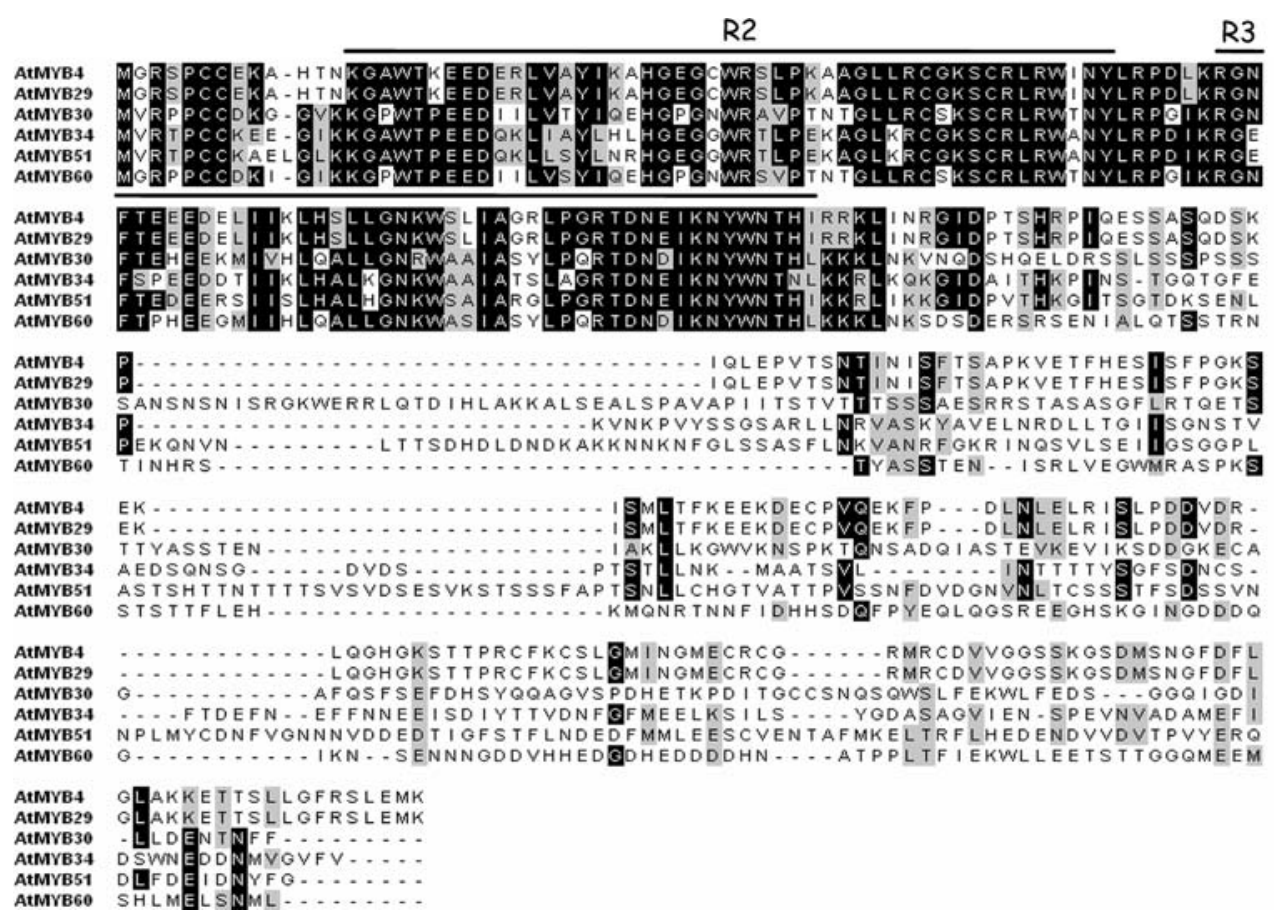




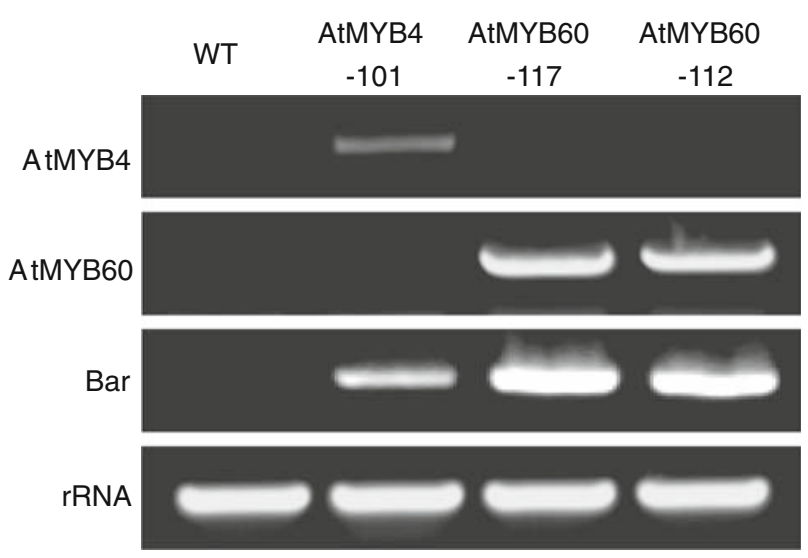

Fig. 3 RT-PCR analysis confirming the transcription of the AtMYB4, AtMYB60 and Bar genes in the indicated transgenic lettuce lines and not in the wild-type (WT) plants. rRNA was used as the control

we compared the morphologies of transgenic lettuce plants overexpressing these genes with that of wild-type lettuce.

More than 20 independent transgenic plants, selected with barstar spray and subsequently confirmed by PCR analysis (data not shown), were obtained with each $A$. tumefaciens strain. There was a large variation in the leaf color phenotypes of these transgenic lines. The AtMYB4101 line showed normal anthocyanin accumulation and a red coloration phenotype, similar to that of the wild-type, but the AtMYB60-117 line showed different levels and patterns of anthocyanin accumulation. Under standard growth conditions, transgenic lettuce plants of the $\mathrm{T} 1$ generation that overexpressed AtMYB60 under the control of the cauliflower mosaic virus (CaMV) $35 \mathrm{~S}$ promoter showed an inhibition (AtMYB60-117) of anthocyanin accumulation (green leaves), compared with that of wildtype plants (red leaves) (Fig. 4a, b). Apart from this inhibition of anthocyanin biosynthesis, no other morphological or developmental abnormalities were detected in this line under standard growth conditions. These results confirm that the exogenous AtMYB60 protein was responsible for the repressed anthocyanin phenotype.

High-performance liquid chromatography (HPLC) analysis of AtMYB60 transgenic lettuce shows that accumulation of anthocyanin compounds is inhibited

To confirm the identity of the anthocyanins that are synthesized in lettuce after transformation with $A t M Y B$ genes, lettuce leaf tissues were harvested and analyzed by HPLC for the presence of soluble anthocyanins. The results confirmed the predominance of cyanidin in the wild-type lettuce leaves, with traces of delphinidin also evident, as we previously described (Park et al. 2007a). The cyanidin and delphinidin peaks were detected in the AtMYB4overexpressing lettuce (AtMYB4-101) and wild-type

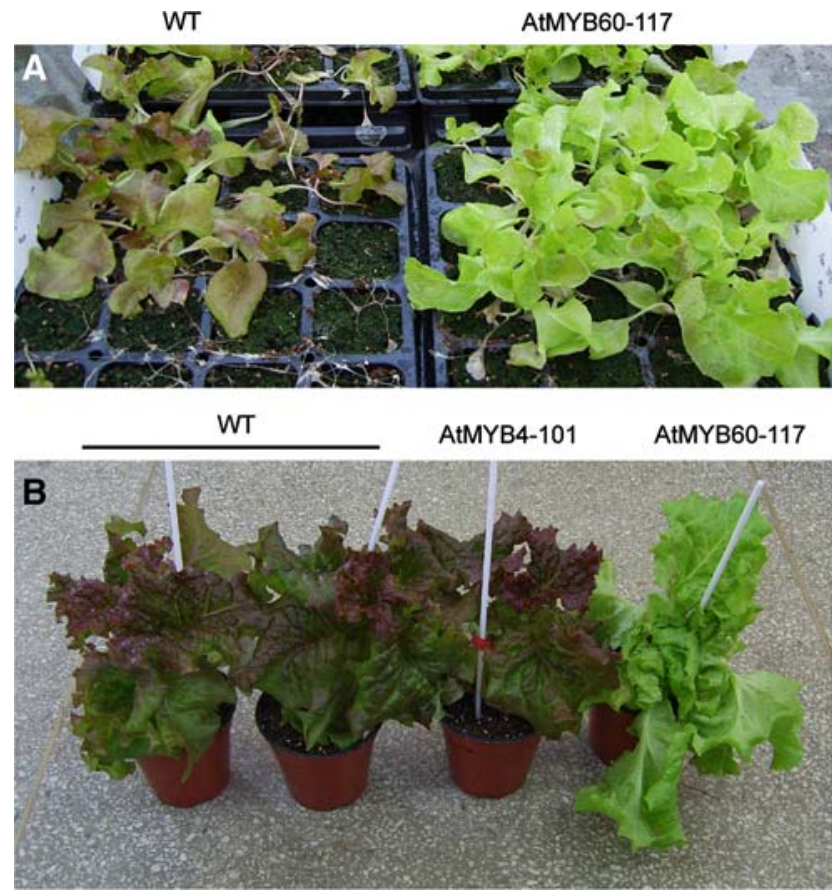

Fig. 4 Morphological analysis showing the inhibition of anthocyanin biosynthesis in lettuce leaves, indicated by the lack of red pigmentation, following the introduction of the Arabidopsis MYB gene, AtMYB60: a young (20 days after sowing) wild-type (WT) lettuce and the AtMYB60 transgenic lettuce line, AtMYB60-117; b morphologies of both the WT and $A t M Y B$-overexpressing lettuce plants at a later stage of growth. Both the AtMYB4-101 and AtMYB60-117 transgenic plants are shown and reveal that AtMYB4 does not inhibit anthocyanin production

lettuce. The AtMYB29-, AtMYB30-, AtMYB34- and At$M Y B 51$-overexpressing lettuce plants that showed a similar red-colored phenotype also produced corresponding cyanidin and delphinidin peaks on HPLC analysis (data not shown). However, clear differences were evident in the patterns of anthocyanin accumulation in the AtMYB60overexpressing lettuce plants (Fig. 5). With HPLC, we found that the cyanidin (red pigment) peak was absent or sharply reduced in AtMYB60-117 and AtMYB60-112. The delphinidin (blue pigment) peak was absent in every AtMYB60-overpxpressing lines. Hence, the anthocyanin derivatives detected in lettuce leaves by HPLC can be correlated with the morphologies of the plants and with the inhibition of anthocyanin biosynthesis by the AtMYB60 protein.

RNA expression profiles of anthocyanin biosynthetic enzymes suggest their coordinated regulation in AtMYB60 transgenic lettuce plants

Based on the correlation between the plant color morphology and our HPLC analysis of the anthocyanins, we undertook to verify the effects of the AtMYB proteins on 


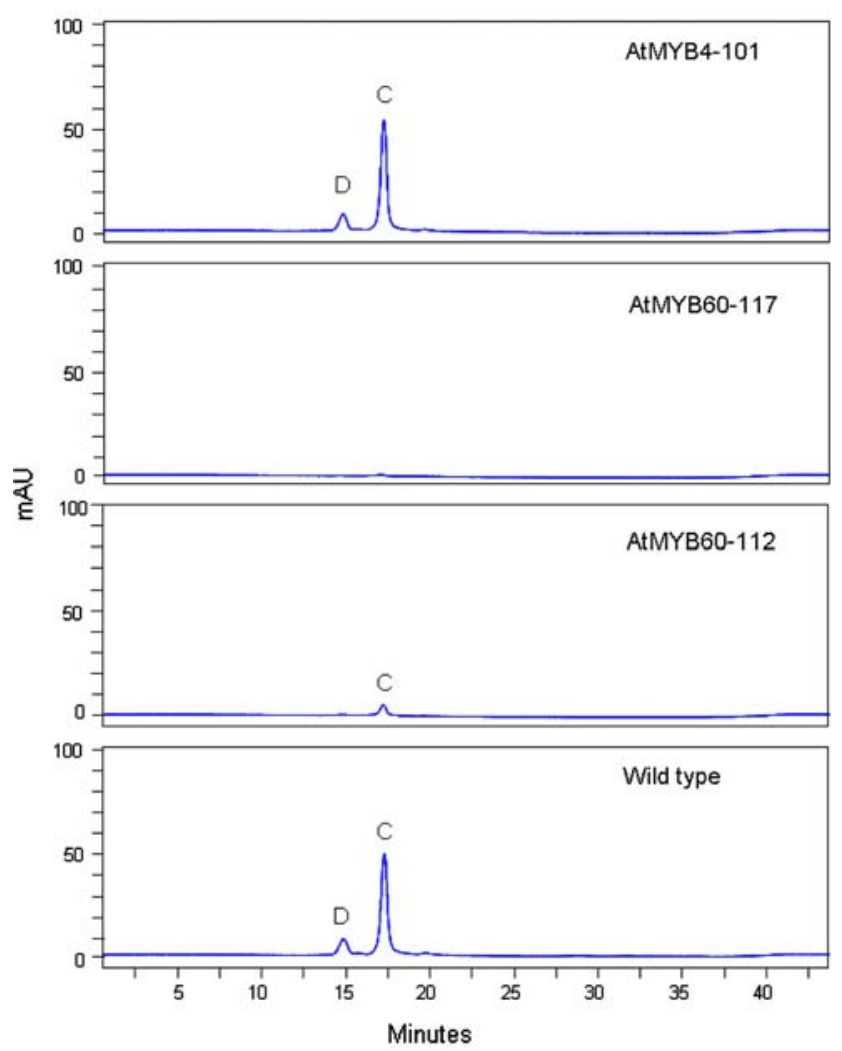

Fig. 5 HPLC analysis of methanolic extracts from both wild-type control (WT) and AtMYB transgenic lettuce leaves (AtMYB4-101, AtMYB60-117 and AtMYB60-112). HPLC chromatograms were recorded at $510 \mathrm{~nm}$. Peak $C$ corresponds to cyanidin and $D$ represents delphinidin. Each chromatogram was generated using the same quantity of injected sample

the expression of the structural genes encoding the enzymes responsible for anthocyanin biosynthesis. As shown in Figs. 4 and 6, a dramatic leaf color change was evident in the AtMYB60-117 (green leaf) and -112 (mosaic phenotype) lines, in which anthocyanin accumulation is inhibited, and these plants showed a strong green leaf color phenotype. The enzymes that act in the anthocyanin biosynthetic pathway have been well characterized in lettuce in our previous study (Park et al. 2007a), in which we also analyzed the expression of the structural genes encoding the relevant enzymes. Following environmental stimuli such as UV-B irradiation, the expression of chalcone synthase (CHS), flavanone 3-hydroxylase $(\mathrm{F} 3 \mathrm{H})$ and dihydroflavonol 4-reductase (DFR) is positively induced, and the accumulation of anthocyanin is increased in lettuce. We also performed RT-PCR analysis using genespecific primers in that study to analyze the expression of these structural genes. They were identified from young UV-B-irradiated lettuce leaves and designated as CHS (CV700441), F3H (CV700152), DFR (CV700105) and UFGT (CV700246) (Park et al. 2007a). The transcript levels of these four anthocyanin biosynthetic genes were compared between the wild-type, AtMYB4- and AtMYB60overexpressing lettuce plants in the present study.

Total RNA extracts were prepared from the leaves of sixweek-old lettuce plants of wild-type, AtMYB4-101 and AtMYB60-117 lines and were analyzed by RT-PCR for the expression of the previously determined structural genes that are involved in the major biochemical pathways of anthocyanin biosynthesis (Fig. 6b). No reduction in the expression of any of these genes was observed in wild-type or AtMYB4101 lines, both of which exhibited red coloration in their leaves. Moreover, the mRNA levels of the $C H S, F 3 H$ and UFGT genes, which catalyze the synthesis of chalcones, flavanones and anthocyanin, respectively, were unchanged in any of the plants. In contrast, the mRNA levels of $D F R$, the production of which catalyzes the conversion of dihydroquercetin and dihydrokaempferol to leucocyanidin and leucopelargonidin (of the leucoanthocyanidins), respectively, was inhibited in the AtMYB60-117 line, which exhibits green-colored leaves with no red pigmentation.

\section{Discussion}

To isolate the MYB-type transcription factor genes involved in phenylpropanoid metabolism, the transgenic expression of six $A t M Y B$ genes was analyzed in lettuce. To date, a large number of transcription factors containing DNA-binding domains that are similar to those of the MYB proteins have been identified across the eukaryotic kingdom (Kranz et al. 1998). Several MYB transcription factors are known to regulate phenylpropanoid metabolism, and the quantities of the various end-products of the associated pathways that accumulate in specific cells, tissues and organs are thought to respresent the balanced expression of several interacting MYB proteins (Tamagnone et al. 1998; Espley et al. 2007). Recently, biochemical studies have also suggested that the MYB-type transcription factors are involved in regulating the branches of the phenylpropanoid metabolic networks in higher plants. Multiple MYBs are also thought to regulate the expression of the structural proteins that drive several downstream pathways of flavonoid metabolism (Moyano et al. 1996). A previous study has also shown that MYB proteins can regulate flavonoid biosynthesis via the transcriptional regulation of the gene encoding $C H S$, an enzyme that catalyzes the first committed step in flavonoid biosynthesis (Feldbrugge et al. 1997).

The lettuce cultivar "Jinjachuckmyun", which we used in our current transformation experiments, shows anthocyanin pigment accumulation in its leaves during development. We have shown in a recent study that environmental stresses upregulate the expression of the genes that encode anthocyanin biosynthetic enzymes in this same 
Fig. 6 RT-PCR analysis of the genes involved in anthocyanin biosynthesis in the leaves of wild-type and AtMYB4- and AtMYB60-overexpressing lettuce plants. The transcripts of four biosynthetic genes involved in general anthocyanin metabolism (CHS, F3H, DFR and $U F G T$ ) were analyzed by RT-PCR in the AtMYB4-101 and AtMYB60-117 transgenic lettuce lines and were compared with the wild-type expression patterns. rRNA was used as the quantitative control

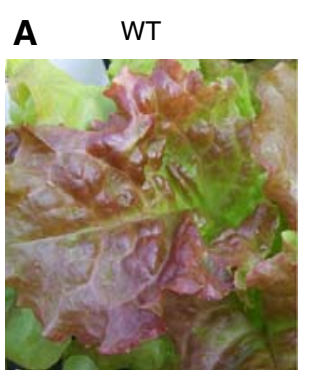

AtMYB4-101

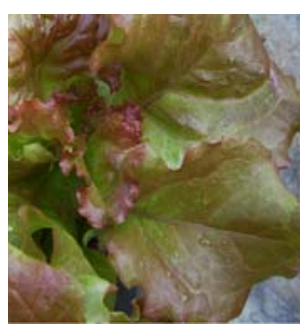

AtMYB60-117

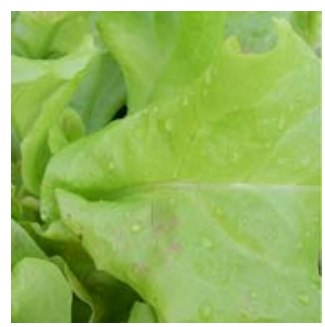

AtMYB60-112

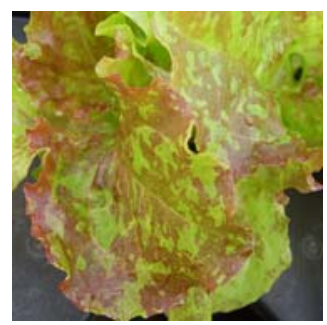

B

$\mathrm{CHS}$

$\mathrm{F} 3 \mathrm{H}$

DFR

UFGT

rRNA
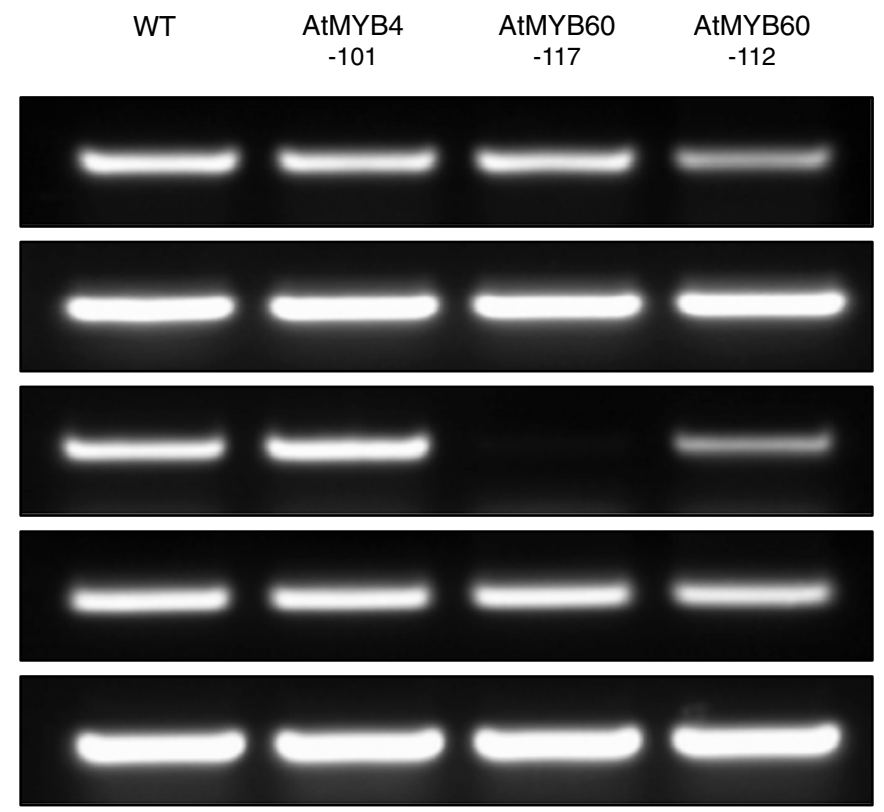

lettuce variety, as demonstrated by their responsiveness to UV-B irradiation (Park et al. 2007a).

The lettuce has merit as a model plant system to further characterize the functions of those gene products in anthocyanin metabolism, because of its leaf color is red under field conditions and green under chamber condition when exposed to UV-B irradiation. The findings described in this study further validate the use of lettuce as a model plant because it offers experimental versatility in terms of its genetic regulation of the inhibition of leaf pigmentation. The function of the MYB proteins as activators (green to red in a growth chamber) or repressors (red to green in the field) of anthocyanin biosynthesis can be characterized directly from a phenotypic analysis of the lettuce leaf color. This system has potential applications as a research tool in a number of areas of plant molecular biology.

We show in our present experiments that the anthocyanin accumulation in leaves is strongly inhibited in AtMYB60 transgenic plants compared with that in wild-type plants (Fig. 4a, b). These results suggest that AtMYB60 functions as a repressor of anthocyanin biosynthesis. Based upon our current findings of the inhibition of anthocyanin biosynthesis and our metabolite analysis of AtMYB60overexpressing lettuce plants compared with the wild-type, we speculate that the synthesis of anthocyanin is principally controlled by the AtMYB60 transcription factor. The production of anthocyanin is inhibited in the AtMYB60-117 and AtMYB60-112 lines, and this is likely to underlie the green leaf phenotype of these plants. Hence, this characterization of the function of AtMYB60 represents a crucial step in furthering our understanding of the molecular regulation of anthocyanin biosynthesis and the transcription of related genes in lettuce and other plants. It is noteworthy in this context that MYB-related proteins generally act as transcriptional activators (Foos et al. 1994).

The R2R3-MYB-related proteins have so far been shown to activate the transcription of structural proteins that act in different branches of the phenylpropanoid metabolism machinery (Martin and Paz-Ares 1997) or that play roles in the plant response to stress signals (Cominelli et al. 2005). Anthocyanin expression in maize is dependent on the presence of both the MYB and MYC types of regulatory factors. It is of some interest, therefore, that in this study, the expression of AtMYB60 alone, under the control 
of the CaMV 35S promoter, was sufficient to repress anthocyanin accumulation in a red lettuce variety.

We also investigated the effects of $A t M Y B 60$ on the expression of the different structural proteins involved in anthocyanin production, which we have identified previously in lettuce. Our results reveal that this MYB protein represses the expression of the DFR gene in the AtMYB-117 and -112 lines. Significantly, DFR is a crucial structural protein that facilitates anthocyanin pigmentation in lettuce and that no such reduction in the expression of this gene was found in the wild-type or AtMYB4-101 lines that also develop red-colored leaves. The DFR protein represents an important branching point within the anthocyanin biosynthetic pathway and catalyzes the commitment step in this process, which is the formation of leucoanthocyanidins from the substrate dihydroflavonol. This indicates that AtMYB60 specifically inhibits the flux of flavonoid intermediates toward the production of leucoanthocyanins by repressing the transcription of $D F R$, with a resulting green leaf phenotype (Fig. 7).

It is interesting to note that the transcriptional response to UV-B irradiation that operates through AtMYB60 focuses on the regulation of $D F R$ expression and anthocyanin accumulation. Recently there has been some debate about the identity of the phenylpropanoids involved in the

\section{4-Coumaroly-CoA + 3 Malonyl-CoA}
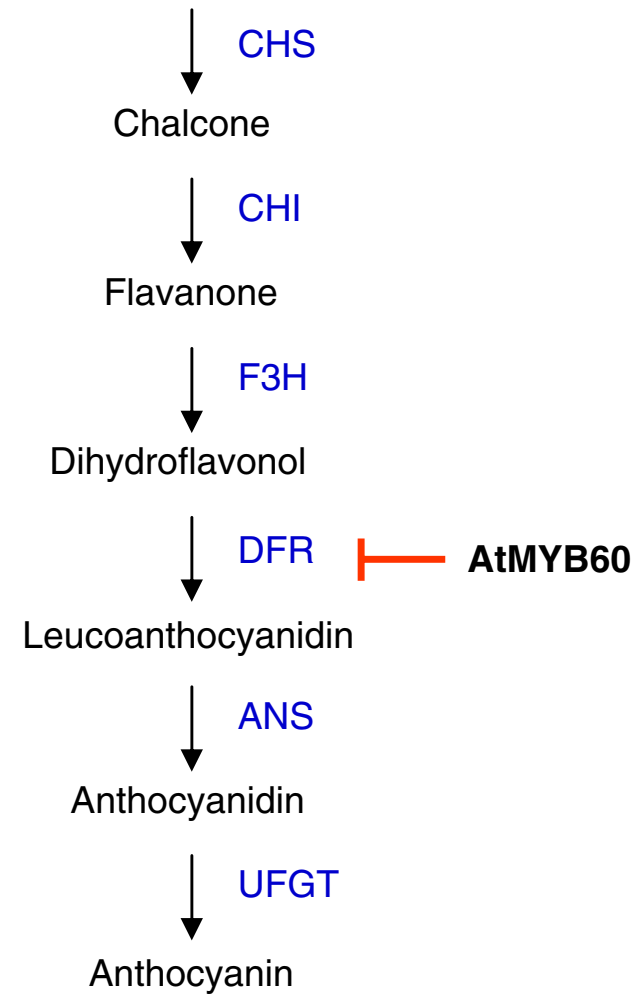

Fig. 7 Schematic representation of the relationship between AtMYB60 expression and the structural proteins that play roles in anthocyanin biosynthesis response to UV-B, which may provide the most effective components of sunscreen formulations. MYB transcription factors that are known to regulate the transcription of genes in the phenylpropanoid biosynthetic pathway have been studied previously (Jin and Martin 1999). The overexpression of PAP1 in Arabidopsis results in the upregulation of the genes encoding phenylalanine ammonialyase (PAL), CHS and DFR (Borevitz et al. 2000; Tohge et al. 2005). Moreover, mutations in the $C H S, D F R$ and LDOX genes reduce the pigmentation of plants (Shirley et al. 1995; Abrahams et al. 2003), suggesting that these proteins are required for the synthesis of both anthocyanidin and proanthocyanidin. Because MYB-binding sites are present in the promoter regions of these flavonoid biosynthetic genes (Nesi et al. 2001; Debeaujon et al. 2003), it is possible that the AtMYB60 transcription factor might also specifically and directly target them. It is also possible that additional target genes that are related to phenylpropanoid metabolism can be negatively regulated by $A t M Y B 60$ when it is overexpressed in lettuce.

The effects of AtMYB60 suggest that this MYB protein acts as a direct transcriptional regulator by binding to the MYB motifs common to the promoters of many structural genes in the same metabolic pathway, thus resulting in the downregulation of this pathway. The precise determination of this regulatory system at the molecular level remains to be achieved. Recently, Cominelli et al. (2005) reported that AtMYB60 is involved in the plant response to stress and its expression is negatively modulated during conditions of drought (Cominelli et al. 2005). A null mutation in AtMYB60 results in a constitutive reduction in stomatal openings and in decreased wilting under water-stress conditions. Therefore, we hypothesize that AtMYB60 has multiple functions in the regulation of anthocyanin biosynthesis and in the plant responses to different environmental conditions, including drought and exposure to UV-B light.

AtMYB60 is the first MYB protein identified that functions as a transcriptional repressor of the lettuce $D F R$ gene in anthocyanin biosynthesis, and thus extends the known roles of the R2R3-MYB proteins in secondary metabolism. Evidence from our transgenic lettuce experiments suggests that the direct repression exerted by AtMYB60 protein produces phenotypic effects in transgenic plants. The engineering of the anthocyanin biosynthetic pathway is potentially of great commercial significance. Because the AtMYB60 transcription factor should act effectively in most plant species, it can potentially be used to control anthocyanin biosynthesis in agriculturally and industrially important plants. This method of modifying the anthocyanin content in plant tissues also opens up new avenues to engineering improvements in commercial crops by altering metabolic pathways via the regulation of different structural genes in these pathways. 
Acknowledgments This research was supported by a grant from a basic research fund (06-011) of the National Institute of Agricultural Biotechnology, the Cooperative Research Project for Bioenergy Crop Development (20070201036015) and BioGreen21 Program (20050501034844) of the Rural Development Administration (RDA), South Korea.

Open Access This article is distributed under the terms of the Creative Commons Attribution Noncommercial License which permits any noncommercial use, distribution, and reproduction in any medium, provided the original author(s) and source are credited.

\section{References}

Abrahams S, Lee E, Walker AR, Tanner GJ, Larkin PJ, Ashton AR (2003) The Arabidopsis TDS4 gene encodes leucoanthocyanidin dioxygenase (LDOX) and is esseential for proanthocyanidin synthesis and vacuole development. Plant J 35:624-636

Borevitz JO, Xia Y, Blount J, Dixon RA, Lamb C (2000) Activation tagging identifies a conserved MYB regulator of phenylpropanoid biosynthesis. Plant Cell 12:2383-2394

Bouche N, Bouchez D (2006) Arabidopsis gene knock out: phenotypes wanted. Curr Opin Plant Biol 4:111-117

Broun P (2004) Transcription factors as tools for metabolic engineering in plants. Curr Opin Plant Biol 7:202-209

Cominelli E, Galbiati M, Vavasseur A, Conti L, Sala T, Vuylsteke M, Dellaporta SL, Tonelli C (2005) A guard-cell-specific MYB transcription factor regulates stomatal movements and plant drought tolerance. Curr Biol 15:1196-1200

Debeaujon I, Nesi N, Perez P, Devic M, Grandjean O, Caboche M, Lepiniec L (2003) Proanthocyanidin-accumulating cells in Arabidopsis testa: regulation of differentiation and role in seed development. Plant Cell 15:2514-2531

Dixon RA, Piava NL (1995) Stress-induced phenylpropanoid metabolism. Plant Cell 7:1085-1097

Espley RV, Hellens RP, Putterill J, Stevenson DE, Kutty-Amma S, Allan AC (2007) Red colouration in apple fruit is due to the activity of the MYB transription factor, MdMYB10. Plant J 49:414-427

Feldbrugge M, Sprenger M, Hahlbrock K, Weisshaar B (1997) PcMYB1, a novel plant protein containing a DNA-binding domain with one MYB repeat, interacts in vivo with a lightregulatory promoter unit. Plant J 11:1079-1093

Foos G, Grimm S, Klempnauer KH (1994) The chicken A-myb protein is a transcriptional activator. Oncogene 9:2481-2488

Harborne JB, Williams CA (2000) Advances in flavonoid research since 1992. Phytochemistry 55:481-504

Holton TA, Cornish EC (1995) Genetics and biochemistry of anthocyanin biosynthesis. Plant Cell 7:1071-1083

Jin H, Martin C (1999) Multifunctionality and diversity within the plant MYB-gene family. Plant Mol Biol 41:577-585

Jin H, Cominelli E, Bailey P, Parr A, Mehrtens F, Jones J, Tonelli C, Weisshaar B, Martin C (2000) Transcriptional repression by AtMYB4 controls production of UV-protecting sunscreens in Arabidopsis. EMBO J 19:6150-6161

Kranz HD, Denekamp M, Greco R, Jin H, Leyva A, Meissner RC, Petroni K, Urzainqui A, Beevan M, Martin C (1998) Towards functional characterisation of the members of the $R 2 R 3-M Y B$ gene family from Arabidopsis thaliana. Plant J 16:263-276

Larkin JC, Oppenheimer DG, Lloyd AM, Paparozzi ET, Marks MD (1994) Roles of the GLABROUS1 and TRANSPARENT TESTA GLABRA genes in Arabidopsis trichome development. Plant Cell 6:1065-1076

Martin C, Paz-Ares J (1997) MYB transcription factors in plants. Trends Genet 13:67-73
Meissner RC, Jin H, Cominelli E, Denekamp M, Fuertes A, Greco R, Kranz HD, Penfield S, Petroni K, Urzainqui A (1999) Function search in a large transcription factor gene family in Arabidopsis: assessing the potential of reverse genetics to identify insertional mutations in R2R3 MYB genes. Plant Cell 11:1827-1840

Moyano E, Martinez-Garcia JF, Martin C (1996) Apparent redundancy in $m y b$ gene function provides gearing for the control of flavonoid biosynthesis in Antirrhinum flowers. Plant Cell 8:1519-1532

Nesi N, Jond C, Debeaujon I, Caboche M, Lepiniec L (2001) The Arabidopsis TT2 gene encodes an R2R3 MYB domain protein that acts as a key determinant for proanthocyanidin accumulation in developing seed. Plant Cell 13:2099-2114

Ohi R, McCollum D, Hirani B, Haese GJD, Zhang X, Burke JD, Turner K, Gould KL (1994) The Schizosaccharomyces pombe $c d c 5+$ gene encodes an essential protein with homology to cMyb. EMBO J 13:471-483

Park J-S, Choung M-G, Kim J-B, Hahn B-S, Kim J-B, Bae S-C, Roh K-H, Kim Y-H, Cheon C-I, Sung M-K, Cho K-J (2007a) Genes up-regulated during red coloration in UV-B irradiated lettuce leaves. Plant Cell Rep 26:507-517

Park J-S, Kim J-B, Hahn B-S, Kim K-H, Ha S-H, Kim J-B, Kim Y-H (2004) EST analysis of genes involved in secondary metabolism in Camellia sinensis (tea), using suppression subtractive hybridization. Plant Sci 166:953-961

Park K-I, Ishikawa N, Morita Y, Choi J-D, Hoshino A, Lida S (2007b) A $b H L H$ regulatory gene in the common morning glory, Ipomoea purpurea, controls anthocyanin biosynthesis in flowers, proanthocyanidin and phytomelanin pigmentation in seeds, and seed trichome formation. Plant J 49:641-654

Pietta PG (2000) Flavonoids as antioxidants. J Nat Prod 67:10351042

Riechmann JL, Ratcliffe OJ (2000) A genomic perspective on plant transcription factors. Curr Opin Plant Biol 3:423-434

Shih C-H, Chu IK, Yip WK, Lo C (2006) Differential expression of two flavonoid 3'-hydroxylase cDNAs involved in biosynthesis of anthocyanin pigments and 3-deoxyanthocyanidin phytoalexins in sorghum. Plant Cell Physiol 47:1412-1419

Shirley BW, Kubasek WL, Storz G, Bruggemann E, Koornneef M, Ausubel FM, Goodman HM (1995) Analysis of Arabidopsis mutants deficient in flavonoid biosynthesis. Plant J 8:659-671

Slamon DJ, Boone TC, Murdock DC, Keith DE, Press MF, Larson RA, Souza LM (1986) Studies of the human c-myb gene and its product in human acute leukemias. Science 233:347-351

Stracke R, Werber M, Weisshaar B (2001) The R2R3-MYB gene family in Arabidopsis thaliana. Curr Opin Plant Biol 4:447-456

Tamagnone L, Merida A, Parr A, Mackay S, Culianez-Macia FA, Roberts K, Martin C (1998) The AmMYB308 and AmMYB330 transcription factors from Antirrhinum regulate phenylpropanoid and lignin biosynthesis in transgenic tobacco. Plant Cell 10:135154

Tohge T, Nishiyana Y, Hirai MY (2005) Functional genomics by integrated analysis of metabolome and transcriptome of Arabidopsis plants over-expressing an MYB transcription factor. Plant J 42:218-235

Vailleau F, Daniel X, Tronchet M, Montillet JL, Triantaphylides C, Roby D (2002) A R2R3-MYB gene, AtMYB30, acts as a positive regulator of the hypersensitive cell death program in plants in response to pathogen attack. Proc Natl Acad Sci USA 99:10179_ 10184

Weisshaar B, Jenkins GI (1998) Phenylpropanoid biosynthesis and its regulation. Curr Opin Plant Biol 1:251-257

Winkel-Shirley B (2001) Flavonoid biosynthesis: a colorful model for genetics, biochemistry, cell biology, and biotechnology. Plant Physiol 126:485-493 